\title{
DESIGNING INTERACTIVE MULTIMEDIA HERITAGE CONTENT FOR MOBILE AND SMALL SCREEN DEVICES
}

Deborah Wilson

$\mathrm{PhD}$ Design Student

Winchester College of Art, Southampton University

1 Crescent Road, North Baddesley,

Southampton

England

debs@,thetalkingwalls.co.uk

http://www.wsa.soton.ac.uk/ \& www.thetalkingwalls.co.uk

Mike Wilman

Southampton Business School

Southampton Solent University

East Park Terrace,

Southampton

England

mike.wilman@solent.ac.uk

http://www.solent.ac.uk

\begin{abstract}
This paper presents an application currently being developed for multiplatform devices, primarily mobile, in which a user can personalise content to suit their interests and learning preference. The Talking Walls ${ }^{\circledR}$ is a pilot project that forms the basis of research regarding an adaptable multimedia template application for heritage sites, museums or historical buildings. It is being designed for users to visually explore information as well as the space they are visiting, learning about the history of a specific place / space / object and how this has changed over time. Metaphorically, a form of architectural digital story-telling for all ages.
\end{abstract}

\section{INTRODUCTION}

The increasing competitiveness of the global tourism industry demands that heritage sites / museums continually seek to add value to attract visitors and the handheld / PDA tour would appear to be a major key in providing this added value. With examples such as the handheld multimedia tour at the Tate Modern and the Natural History Museum (BARRY 2005-6) consumer use of handheld devices is increasing. The development of content for these devices is an area that is of increasing importance to both the designer/creator and the consumer. It is not enough to re-purpose content already provided in other media forms, the screen size dictates new form and new content to be designed specifically for the small screen (Moll 2005).

Children are also very important in the way the content is developed, they have / are growing up with not just computers but mobile devices and social networking, the anytime, anyplace, anywhere 'Martini' generation (Ranchhod 2007). They will be the market segment that is likely to know exactly what they would like on their mobile devices, how they would like to learn and what is likely to keep them using the devices for more than just communication. Although, according to cellphone tracking company 
M:Metrics, (PR.Newswire 2007) statistics show that 77\% of people aged 55-64 have mobile phones. Where it might have been assumed that this technology would only appeal to the younger generation, these figures show that this may not be the only market to design for. The 55 - 64 age range may have more in the way of a disposable income, combined with possibly less responsibilities and more time to explore their cultural heritage, which would also make them a quite important sector to consider (Petrecca 2007).

In the design process, consideration for the type of device (mobile or PDA), how it will be used (touch screen or keypad), and how the content is delivered (wireless, RFID or storage cards) will hugely affect consumer acceptance of the content and the technology involved. If any part of the exploration process or use of the device and /or content lacks an intuitive approach the consumer is likely to falter and have an incomplete or bad experience, resulting in a reluctance to use it again (Braiterman and Becker 2008).

The questions that have arisen when talking with managers / owners of heritage sites in previous interviews have been 'will the visitor accept the use of these handhelds?' and 'will they know how to use them?' (Wilson 2002-2006) A few years ago, the answer would have been no, accept for a few who liked to be at the cutting edge of technology. This has changed quite considerably. In 2007, the smartphone industry increased by $44 \%$ to approximately 24.5 million units with the PDA units increasing by $54 \%$ in 2007 (Nielssen 2007) showing that acceptance is growing rapidly and becoming a recognised method for use within museum and heritage sites (Aoki and Woodruff 2000). This does not really seem to be where the issue lies for the overall success of these models, it lies more with the content and user behaviour.

In order to understand what content might be acceptable, it is necessary to research user behaviour, how they might like to use the content, what type of content is going to engage them and encourage them to explore further and how they will interact with this information on a small screen.

These questions form part of ongoing research for the current development of a prototype called The Talking Walls ${ }^{\circledR}$. The Talking Walls ${ }^{\circledR}$ is a multimedia template application that is being designed to be adapted to suit any heritage site, museum or historical building. Users will be able to visually explore and navigate where they are visiting via a mobile phone / handheld device, or a kiosk / DVD / website and learn about the history of a specific place / space / object and be able to see how this has changed over time. It is a form of digital story-telling (Miller 2004) with the history / context of the building or object forming the basis of the content, with additional entertaining and educational elements to help provide a multimedia visually-rich resource for all ages.

\section{EDUCATIONAL DEVELOPMENT FOR CHILDREN}

The concept has been devised from the need to provide children with visually rich 3D material on which their imagination can feed and expand. As in the case of Dunster Castle, the visiting school children are faced with a fairy tale manor house set on a beautiful wooded tor a few miles from the sea. They are then asked to imagine this as a moor covered tor and hill fort with the sea lapping the tor base. This is quite a leap of imagination, but if given virtual images / animation of what it was like and to be able to 'walk-through' this environment, the task becomes easier and their imagination is fired 
up. This was the original task given by a volunteer guide in 1995, and forms the background to the development of the The Talking Walls ${ }^{\circledR}$ multimedia application.

This need to provide children (and adults) images on which to feed their imagination is still relevant today. For thousands of years, storytelling has been the main method of learning, passing on knowledge from one generation to another either verbally, pictorially or written. Once the print process was invented, the same knowledge was converted into books, some beautifully illustrated to enhance and create emotion with the content. For children, from the late 1800s comics were introduced and became a popular form of entertainment and at the same time stimulating their minds with story telling through pictures (an early form of today's mobile snacking?). With the more recent personal computer technology, CD-ROM's and other forms of interactive content, games and puzzles became accepted methods of passing on this knowledge and engaging the user. Now with Web (1.00 \& 2.00), the information and knowledge base has exploded and is available to anyone who has a computer and / or mobile phone (Braiterman and Becker 2008).

For the majority of this time, people visited heritage sites, initially to see how other people lived and then to learn a little of their own culture, (Poria, Butler et al. 2004) (Masberg and Silverman 1996). The information provided at these sites has increased considerably with the need to attract and educate visitors, creating learning environments as well as somewhere to relax and explore with family / companions on a day out. The visitor's thirst for historical and cultural knowledge arrived hand in hand with the increase in technology and their subsequent expectation of being able to access information wherever they are, at any time (Hooft 2006).

There have been different methods given to feed this 'thirst' ranging from guidebooks (separate ones designed for children), to static period displays, seek and find sheets, exhibitions, kiosk displays and more recently, web sites, some specifically aimed at children containing cartoon-like images, puzzles and games. These seem to be aimed primarily at a very young age range. The older child does not seem to be so well catered for, and although they might be interested in a more adult interpretation, the material on offer may not enthuse or involve them enough to learn more. An application that will allow any user to explore areas such as creative writing, art, mathematics, design and technology, geography and communities through which they will also learn about history and culture would be ideal from an educational perspective. It would also need to entertain and engage them, so perhaps the information would need to be provided via puzzles, games and story-telling, information they can interact with and become engaged. The Talking Walls ${ }^{\circledR}$ concept has this as it's prime goal and to also cater for mixed abilities and ages by being able to pick and mix from the content to suit their level and interest.

\section{INVOLVEMENT AND ENGAGEMENT}

It has already been pointed out that a variety of users, from children to older adults, will be using Talking Walls applications in a variety of contexts and ways. The way they will want to use the technology will vary not just in terms of their age, but their interest in mobile devices and their desire to use them for learning purposes.

Firstly, the level and depth of involvement will vary. Laaksonen (1994) considers that there are three sides to involvement:

[1] Cognitive perspective - is the perceived personal relevance of the item that is 
important to the consumer

[2] Predisposition to act - the interest or level of emotional attachment, arousal,

drive or motivation evoked by a stimulus - for example, the level of interest in the subject and the interest triggered by the potential for learning and variety that the user perceives in the application

[3] Response view - the reaction of an individual to a stimulus, which affects the 'learn-feel-do' sequence

User interaction patterns will therefore vary according to a number of factors and it will be important for museums or galleries (hosts) to be aware of the wide range of use levels to which the application will be put, and that this will not just a simple case of age. Some users may need some initial hands-on training before they are familiar with the technology.

In an article for a seminar Jennie Fordham, Education Officer at English Heritage / HHA wrote: 'Learning is a process of active engagement with experience...Effective learning leads to change, development and the desire to learn more. It is all about involvement - if we are involved, we care, and this has to start with understanding... through learning at whatever age.' (Fordham 2005)

Visiting an historic house / museum already places the user in a rich contextual environment. They can interact with their surroundings by looking, listening and touching. They can converse and learn from the objects and people around them but what will actually engage them and lead them to want to discover more either while they are still onsite or once they have left the site?

Interaction and entertainment are considered to be prime methods for engaging and involving users. Interactivity is what makes the digital media, whether on the PC, console or television very different from other media such as books, films and television programmes, it is the interaction of the user with the content that engages the user. The user is no longer being passive, listening to the audio or watching the video clip, they are actively changing their experience, making it their own, personalising and becoming involved. Each user creates their own experience, learning different skills and through choosing different characters, their storytelling imparts different information, or possibly the same as another, but in a way that is pertinent to their level of understanding and learning preference. This is the basis on which games are designed, although primarily as a form of entertainment, knowledge is still acquired through the story of the game.

Game playing has proven to be a great success with mobile phone users earning considerable revenue for the mobile industry. The ability to play a game at any time, any place and anywhere (snacking) helps in this success, but also the designers have discovered that designing games specifically for the mobile phone instead of 'shoehorning' console games has increased in popularity becoming the 'cornerstone' of the mobile gaming industry (Loader 2006). This example perhaps gives an insight into how it might be possible to create a similar success for designing heritage content that can be used on a small screen. Perhaps the design needs to encompass small 'snack' sized chunks (Moses 2007) of engaging information? Perhaps created from moving visual imagery that tells a story? Perhaps allowing the user to interact, re-use and re-mix, generating their own chunks of information?

\section{USER INTERACTION}


This creates considerable problems for the designer. No longer can the designer create content and dictate the path of the user, this would make it a passive experience, the user would have nothing to do but sit back and watch what happens, or at the very least, click the buttons indicated for another static image. There is not a great deal of difference between this and turning the page of a book or switching channels on a television, it is not interaction in its full sense. The success of Web 2.0 has proven that users want to generate their own content and are doing so extremely well, seemingly without help from designers, or is this truly the case? If the web site has not been designed in the right way, would the user be able to interact with it and post, create and re-generate their own content? The designer has provided a template in which this can happen, i.e. YouTube's website. How then, can designers transpose this to a small screen and provide the user with a model they can interact with and re-generate content? A template needs to be created that will allow this to happen, providing access to a database of generic / specific content which they can then re-model but through a clear, simple and easy to navigate 'small screen' design.

The Talking Walls ${ }^{\circledR}$ model is being designed to create this type of experience as much as possible, to entertain, engage as well as inform. The content will be a growing database of 3D objects of different historical periods, images, film, virtual environments, educational puzzles, characters, historical fact sheets and lifestyle images / video. A visually rich resource that the user will be able to choose from and add to. It is envisaged that children and adults will walk around a property or museum discovering more about the history, life and social impact of the place by using / interacting with the content on a PDA provided by the site or their own smartphone. Parents / adults would be able to explore the areas they are interested in, knowing that their children are also involved at their own learning level. Similar to gaming, The Talking Walls ${ }^{\circledR}$ database will contain a range of character identities with their own narratives. These will remain constant through time and act as guides which the user can choose to be their virtual companion for the duration of their visit. The characters are dressed in the period of the relevant time slice and relate stories of the period imparting tacit knowledge of the period and history of the site.

A choice of animated walkthrough routes are also provided using the present, or previous owners as guides to show you round their home. These guides provide additional information about the rooms / space / objects the users are visiting and will make the virtual experience more personal by being tailored to their own interests. The walkthroughs will enable people with limited mobility to enjoy and participate in exploring the garden/ house / space, in areas they may not be able to easily visit. The user would be able to create their own points of interest as they navigate around the site, building a more personalised tour which they can expand upon at a later date, possibly online.

\section{DYNAMIC INTERFACE}

The KubeMatrix ${ }^{\mathrm{TM}}$ is the planned interface to all of this content. It is a unique concept acting as a navigational tool, representing time and space, content and links or objects and connections in a 4-dimensional cube matrix structure, as the interface exploring the content and allowing the user to choose their age range and content. The user will then be able to track their progress, with the KubeMatrix ${ }^{\mathrm{TM}}$ indicating the "spaces" they have visited and the routes taken. During a tour each cube of the KubeMatrix $^{\mathrm{TM}}$ represents a room / object or other designated space, and the connecting 
tubes are the passageways, connections or pathways between. The vertical layers are the different eras that are important to the history of the site, or the different hierarchies or links to content or objects of that time. Users will be able to see how the different eras impacted on day to day life by jumping from a room in one era to the same room in another era.

Children will be able to grasp their historical background much more easily and have an element of fun in doing so. The cubes and layers can be used as content holders with the interconnecting links creating a visual map of how the content is related and how to navigate between it. The KubeMatrix ${ }^{\mathrm{TM}}$ will also work with GPS, allowing the client site to track the areas most / less visited, and record the user's visit. The recording could then be sold back to the user as a memento of their day, and / or be stored for future visits. Should the user return, they would be able to track the areas already visited and explore new areas. They will be able to feed back to the database via the website.

The prototype has been tested with varied audiences ranging from individuals to small groups with good feedback and several comments on how easy it was to use. The templated interface has been designed to be simple, very visual and intuitive to use. This has been based on the web design general rule of thumb being three clicks to get to your destination within the site, this paradigm should follow for any screen based application, allowing easy and quick navigation through the content (Nielsen 2000).

\section{HUMAN INTERFACE}

Generally, theories around the diffusion of innovation seek to explain and predict adoption rates. Factors that have been found to influence adoption rates include:

\section{Adopter characteristics \\ Social networks \\ Communication process \\ Promoter's strategies \\ Innovation attributes, e.g. usability}

And thus Constantiou, Damsgaard \& Knutsen (2007) suggest that it is not only critical to identify core characteristics of adopter types, but also to determine the various categories of users based on their behaviour patterns and how they perceive a technology application might be of value. For example, a casual visitor to a museum may not wish to take the time to work out how a visual tour could be of benefit, whereas someone with a keen interest in art might tour a gallery exhibition and take time to learn the application in order to extract the information and the benefits they require.

Constantiou, Damsgaard \& Knutsen further believe that a learning perspective should be taken towards users and categorise mobile users according to four types talkers (who use mobile voice applications only); writers; photographers and surfers. The adoption of technology and its services will evolve over time as people learn. Social networks are very important in this regard. Early adopters will discuss it with late and laggard adopters and educate each other about the possibilities afforded by the application.

In contrast, Sarker and Wells (2003) state that factors affecting the implementation and acceptance of wireless technology are interface characteristics and network capabilities. Both are crucial in any application in a heritage situation.

In particular, Sarker and Wells believe that key factors affecting usage are: 
Age of the user-adopter

Technology self-efficacy, i.e. the individual's confidence in using devices

Cultural origin e.g. some users may view a visualisation as trivializing a serious subject

Interface characteristics - a device and its application must be easy to use

Network capability - reliability is essential, including immediacy and the ability to search quickly for the specific information required

\section{CONNECTIVITY}

With regards to connectivity, The Talking Walls ${ }^{\circledR}$ is being designed to work via wireless technology or by downloading to a storage card prior to visiting the site. Both methods would allow the user to freely roam around the site and allow the user to have a degree of interaction with the content and the site / exhibits. This also allows the user to choose what they would like to explore and the type of content with which to do this. This is very important for the user, especially those that are used to mobile technology and like to control the amount of information they are interacting with. If they do not have a suitable phone or handheld device, the basic content can be supplied already downloaded to a wireless PDA / handheld device which the user would hire on site. The additional content for this method would be able to be downloaded as they are exploring. They would then be able to purchase this storage card and continue to extend the experience, exploring in further detail chosen areas via the website on their home computer.

A popular method of providing information to the handheld device has been via RFID tags. This ties the user to being in front of the object until the information has been given. Not only this but they may also be impractical in some circumstances, i.e. a user may walk past an object and the information is pushed to them even though they have not chosen to stop at this exhibit.

The user may also need to be reasonably near the exhibit for the information to flow, which again, may not be practical i.e. the exhibit is roped off or too high such as paintings on ceilings and borders (Aoki and Woodruff 2000). Most importantly, the information is a one way flow; interaction does not exist in this method, the user will not be able to generate their own information back to the tag, perhaps only back to the web site if the handheld device is wireless enabled. From a user's perspective, this method may be the most frustrating with regards to content flow and interaction and not one to use as the main source of technology with the Talking Walls ${ }^{\circledR}$. It may be that a combination of technologies will be the answer to providing a seamless interaction with the device, the site and the user. Any attempt at providing an application that is difficult to use, unreliable or does not provide the user-driven data promptly is unlikely to be popular. Users must feel confident that, as they find their way around a museum or gallery, the technology is a genuine learning aid that will greatly enhance the quality of their visitor experience, seamlessly.

The aim of the initial pilot project will be to test both the theory, the design and the technology from a consumer's perspective. This pilot is currently under development and is the object of a SEEDA funding bid covering the recreation of the Cistercian Abbey at Beaulieu in the New Forest, Hampshire. The abbey was partially destroyed during the Reformation, the dormitories and cloisters being all that remain so the pure majesty and magnificence of how the building dominated Beaulieu is now lost. The exterior of the Abbey will be shown in fully rendered three dimensional form with the 
ability to 'fly around' the structures using the mouse or pointing device of the computer equipment (hand-held, mobile, kiosk screen, desktop or laptop computer). It will be possible to enter the interior of the main buildings, 'walk through' them, look up, down and around, and zoom in to view specific features such as: stone carvings, windows, doors, altar pieces and other fixtures.

By creating this imagery and making it possible for the user - of any age to explore the Abbey as it once looked, it is hoped they will be engaged and want to explore further. The children and how they can be motivated has always been the primary cause for developing a heritage application such as The Talking Walls, so the additional elements of puzzles, cartoon characters and story telling are particularly important to include. The history and visualisation of the inhabitants' lifestyles will hopefully bring to life their culture as well provide a learning tool for visitors to Beaulieu Abbey whether they are children, students or adults, whatever age.

\section{References}

[1] AOKI, P.M. and WOODRUFF, A.: Improving Electronic Guidebook Interfaces Using a Task-Oriented Design Approach. Museum and the Web 2000 .

[2] BARRY, A.: Engaging with a larger, more diverse audience. Annual Review, Natural History Museum.

[3] BRAITERMAN, J. and BECKER, M.: "Academic Review: Customer Experience and Mobile Marketing." Jared Research / iLoop Mobile.

[4] CONSTANTIOU, I. DAMSGAARD, J. and KNUTSEN, L.: The Four Incremental Steps Toward Advanced Mobile Service Adoption. Communications of the ACM, Vol 50 No.6

[5] FORDHAM, J.: What is learning all about - why does it matter? HHA Seminar

[6] HOOFT, M.: Ubiquitous Computing: More than Handhelds. HandHeld Learning 2006, London

[7] KARDES, F.: Consumer Behaviour and Managerial Decision Making. Addison Wesley Longman Inc.

[8] LOADER, J.: Entertaining Mobile. Mobile Entertainment Markets Opportunities and Forecasts 2006-2011. Juniper Research.

[9] MASBERG, B.A. and SILVERMAN, L.H.: "Visitor Experiences at Heritage Sites: A Phenomenological Approach." Journal of Travel Research.

[10] MILLER, C.H.: Digital Storytelling - a creator's guide to interactive entertainment. Oxford, Focal Press, Elsevier.

[11] MOLL, C.: Mobile Web Design: Methods to the Madness. Mobile Web Design.

[12] MOSES, A.: 'Snacking' to spark mobile video explosion. The Age.

[13] NIELSEN, J.: Designing Web Usability. Indianapolis, New Riders Publishing.

[14] PETRECCA, L.: Tech giants target older buyers - and their cash. USA Today.

[15] POIRA, Y.R. et al: Links between Tourists, Heritage, and Reasons for Visiting Heritage Sites. Journal of Travel Research.

[16] PR NEWSWIRE: M:Metrics Study. News and Information

[17] RANCHHOD, A.: Developing Mobile Marketing Strategies. International Journal of Mobile Marketing.

[18] SARKER, S. and WELLS, J.: Understanding Mobile Handheld Device Use and Adoption. Communications of the ACM Vol 46 No 12

[19] WILSON, D.: The Talking Walls Research. Masters in Interactive Production 